\section{Surgical removal of choroidal neovascular membranes after laser photocoagulation for diabetic maculopathy}

\begin{abstract}
Purpose Choroidal neovascularisation (CNV) occurs rarely following laser photocoagulation for macular oedema in diabetic retinopathy, and its management is not well established. We report the clinical course and visual outcomes in a series of patients who underwent surgical extraction of the CNV membrane.

Methods A retrospective review of 4 cases was carried out.

Results Two women and 2 men, mean age 59.5 (range 58-62) years, were reviewed. The CNV developed 2-24 (mean 11) months after laser coagulation and resulted in decreased visual acuity to between $6 / 60$ and HM. All underwent pars plana vitrectomy, extraction of the CNV membrane and fluid-air exchange. Follow-up ranged between 9 and 48 months. In 2 patients, the vision improved by 4 and 1 Snellen lines respectively and remained stable, in 1 patient it improved by 1 line initially but then regressed to $C F$, and in 1 patient it remained unchanged. Recurrence of CNV occurred in only 1 patient. Histological characteristics were those of $\mathrm{CNV}$ without evidence of photoreceptors.

Conclusions This study shows that surgical removal of post-laser $\mathrm{CNV}$ is technically feasible despite the previous laser scars and may have beneficial outcome. This surgical approach may provide a therapeutic option in such patients.
\end{abstract}

Key words Choroidal neovascular membranes, Diabetic retinopathy, Vitrectomy

Choroidal neovascularisation (CNV) is a common cause of severe visual loss and may occur in many conditions affecting the choriocapillaris, Bruch's membrane and retinal pigment epithelium (RPE) ${ }^{1}$ In age-related macular degeneration (AMD), the most frequent cause of $\mathrm{CNV}^{2}$ the neovascular membrane appears to result from diffuse changes in Bruch's membrane, whereas other cases arise from more focal pathological processes. Inflammatory conditions, ${ }^{3}$ resulting in choroidal or chorioretinal scarring, or accidental or surgical trauma ${ }^{4}$ including laser photocoagulation, ${ }^{5}$ have also been reported to cause $\mathrm{CNV}$.

The value of laser photocoagulation in the treatment of extrafoveal CNV has been established by randomised trials, ${ }^{6,7}$ but the management of subfoveal CNV remains controversial. Macular laser photocoagulation is associated with development of a scotoma in the area of laser and therefore loss of central vision if used to treat subfoveal membranes. ${ }^{8}$ Surgical extraction of subfoveal CNV, in an attempt to preserve central vision, has been found to be helpful in some cases, ${ }^{9}$ particularly presumed ocular histoplasmosis syndrome (POHS), ${ }^{3}$ but less so in AMD. ${ }^{10}$

We report the clinical findings in 4 patients with subfoveal CNV following macular laser photocoagulation for diabetic retinopathy, who underwent subretinal surgery for extraction of the neovascular membrane, and the histological findings in 2 of these cases.

\section{Materials and methods}

A retrospective review was performed of the medical records of 4 patients who underwent surgical extraction of a subfoveal choroidal neovascular membrane arising following grid macular laser photocoagulation. All patients underwent full ophthalmic examination, including slit-lamp biomicroscopy, fundus photography and fluorescein angiography. We reviewed the pre-operative and post-operative clinical details, and evaluated the fundus colour photographs and fluorescein angiograms.

All 4 patients underwent a standard approach as described by Thomas and Kaplan. ${ }^{3}$ Briefly, a pars plana vitrectomy was performed with removal of the posterior vitreous cortex. A posterior retinotomy site was created to allow access to the choroidal neovascular membrane. A subretinal pick was used to mobilise the area at the edge of the neovascular complex.
I.G.M. Duguid

Z.J. Gregor

Moorfields Eye Hospital London EC1V 2PD, UK

\section{P.E. Rubsamen}

Bascom Palmer Eye Institute University of Miami School of Medicine

Miami, FL 33136, USA

Y. le Mer

Service d'Ophtalmologie Centre de Hospitalier André Gregoire

93105 Montreuil, France

P. Luthert

Department of Pathology Institute of Ophthalmology University of London London EC1V 9EL, UK

Mr I.G.M. Duguid Moorfields Eye Hospital City Road

London EC1V 2PD, UK

Received: 25 April 2000 Accepted in revised form: 27 September 2000 


\begin{tabular}{lllll}
\hline Patient no. & $\begin{array}{l}\text { Pre-operative visual } \\
\text { acuity }\end{array}$ & $\begin{array}{l}\text { Best post-operative } \\
\text { visual acuity }\end{array}$ & Follow-up (months) & Post-operative angiography findings \\
\hline 1 & $6 / 60$ & $6 / 36$ & 12 & No recurrent CNV; mild RPE atrophy \\
2 & $3 / 60$ & $3 / 60$ & 9 & No recurrent CNV; extensive RPE atrophy \\
3 & $6 / 60$ & $6 / 12$ & 24 & No recurrent CNV; mild RPE atrophy \\
4 & HM & $3 / 60$ & 10 & Recurrent CNV \\
\hline
\end{tabular}

$\mathrm{HM}$, hand movements; $\mathrm{CNV}$, choroidal neovascular membrane; RPE, retinal pigment epithelium.

Following this, the infusion pressure was raised in the eye so as to prevent subretinal bleeding and then subretinal forceps used to remove the CNV. After a $5 \mathrm{~min}$ period of observation, the intraocular pressure was then lowered progressively ensuring no further bleeding occurred, and a fluid-air exchange was performed. Patients were asked to remain in a face-down position for 3-5 days following surgery.

\section{Results}

Four patients with adult-onset diabetes mellitus developed choroidal neovascularisation following laser photocoagulation. There were 2 women and 2 men with a mean age of 59.5 (range 58-62) years. Follow-up ranged from 9 to 48 months. The CNV developed an average of 11 (range 2-24) months following laser and resulted in reduction of the visual acuity to between 6/60 and HM (Table 1). Each patient had a large subfoveal choroidal neovascular membrane at the time of surgery.

At surgery 2 of the patients had neovascular membranes that were thought to be overlying the retinal pigment epithelium. In the third patient (case 2) the neovascular membrane was found to have been adherent to several areas of laser scarring. The nature of the membrane of the fourth patient was not described. In patients 1 and 3, post-operative visual acuity improved by 1 and 4 Snellen lines respectively. The vision in patient 4 improved initially by 1 line and then deteriorated to the pre-operative level, and in patient 2 remained unchanged (Table 1). Choroidal neovascularisation recurred in patient 4 after 2 months.

\section{Clinical details}

\section{Case 1}

A 58-year-old Caucasian man presented with a 4 month history of decreasing left visual acuity. Six months previously he had undergone focal laser photocoagulation for clinically significant diabetic macular oedema associated with non-insulin-dependent diabetes mellitus of 8 years' duration (Fig. 1a, b).

At presentation his right visual acuity was $6 / 24$ and the left visual acuity was $6 / 60$ eccentrically. In the right eye there was advanced diffuse macular oedema with intraretinal haemorrhages and a subfoveal plaque of exudate. In his left eye he had serous macular detachment and a subretinal haemorrhage as well as numerous intraretinal microaneurysms, exudates and cystoid macular oedema (Fig. 1c). Fluorescein angiography disclosed a left choroidal neovascular complex apparently associated with a large laser scar. There was diffuse intraretinal leakage in both maculae (Fig. 1d).

Following a thorough explanation of the therapeutic options he elected to undergo surgical removal of the left subfoveal choroidal neovascular complex. At surgery the $\mathrm{CNV}$ could be mobilised and removed relatively easily leaving mottled retinal pigment epithelium in situ. Postoperatively his serous retinal detachment resolved though the diffuse macular oedema and cystoid changes remained, reducing the left visual acuity to $6 / 36$ (Fig. 1e). He declined any further laser treatment. Histological examination showed a typical CNV membrane with multiple vascular channels and an attenuated layer of retinal pigment epithelium partially lining one surface (Fig. 1f).

\section{Case 2}

A 58-year-old woman with adult-onset diabetes mellitus of 20 years duration was first seen in July 1995 when she was found to have clinically significant macular oedema in the right eye. Macular laser photocoagulation was performed using a $50 \mu \mathrm{m}$ spot-size and argon blue-green laser. Pre-laser visual acuity was 6/18 and following laser the macular oedema resolved with the visual acuity remaining unchanged. In August 1996 she presented with further deterioration of vision. At that time she was found to have visual acuity of $3 / 60$ with clinical evidence of subretinal neovascularisation. Fluorescein angiography showed a large, sea-fan-shaped CNV which appeared to be emanating from a supertemporal area of prior laser photocoagulation. The membrane was greater than 4 disc diameters in size and was subfoveal

(Fig. 2a, b).

The patient underwent surgical removal of the neovascular membrane and it was noted intraoperatively that the neovascular membrane was located in the subretinal pigment epithelial space and was adherent to the overlying RPE in multiple areas where previous laser scars were present. Removal of the neovascular membrane was possible, but the overlying retinal pigment epithelium was denuded at the time of surgery.

Post-operatively the visual acuity remained $3 / 60$. The post-operative fluorescein angiogram confirmed loss of the retinal pigment epithelium (Fig. 2c, d). 


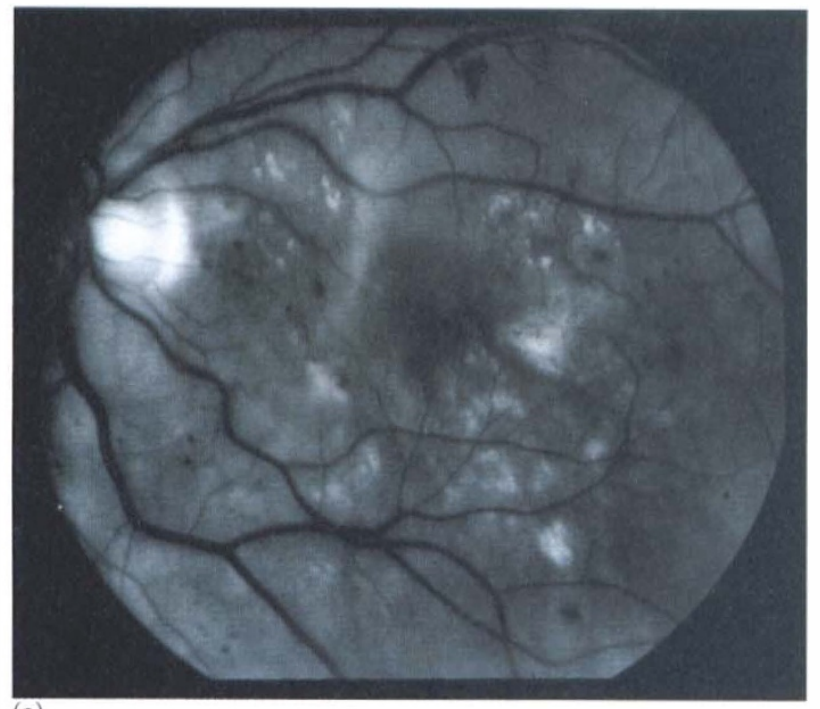

(a)

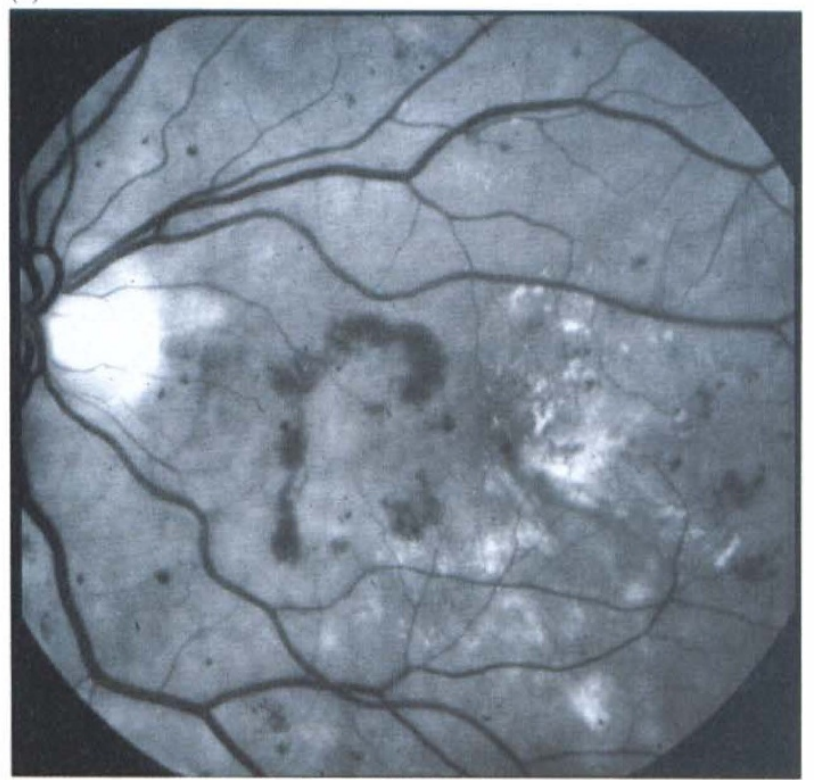

(c)

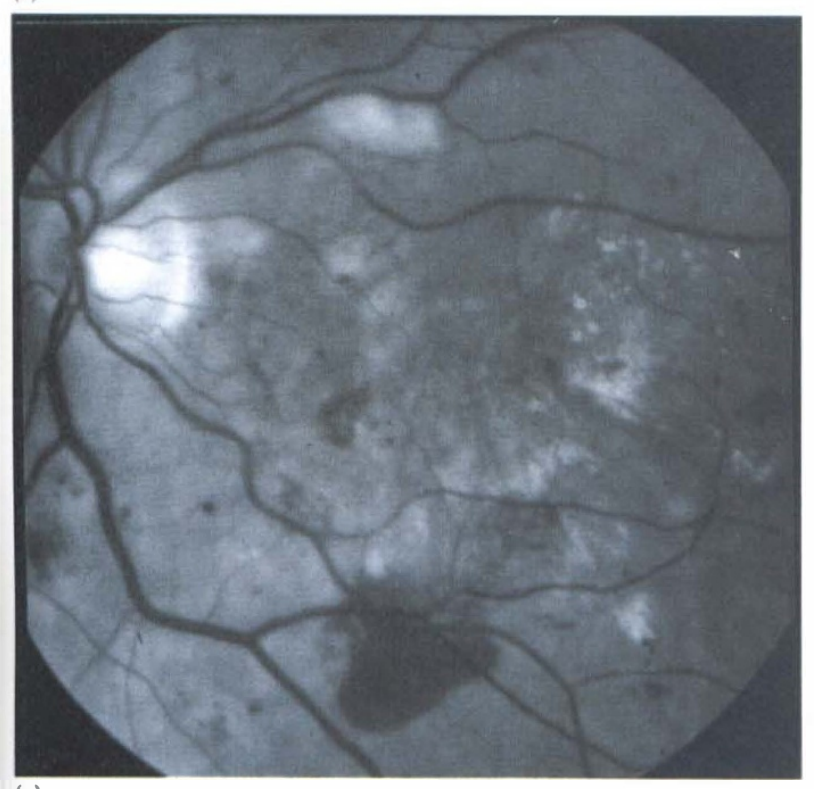

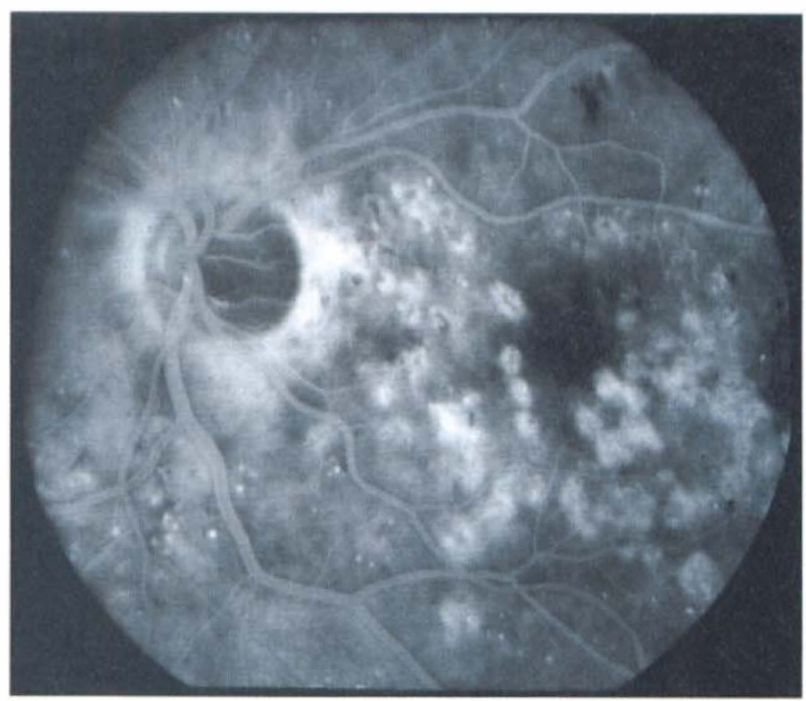

(b)

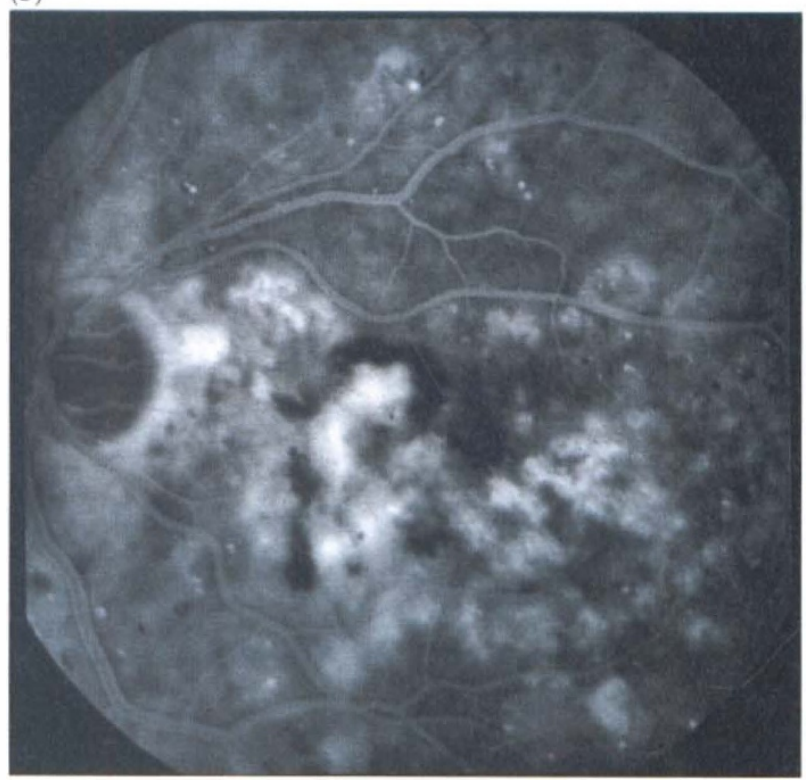

(d)

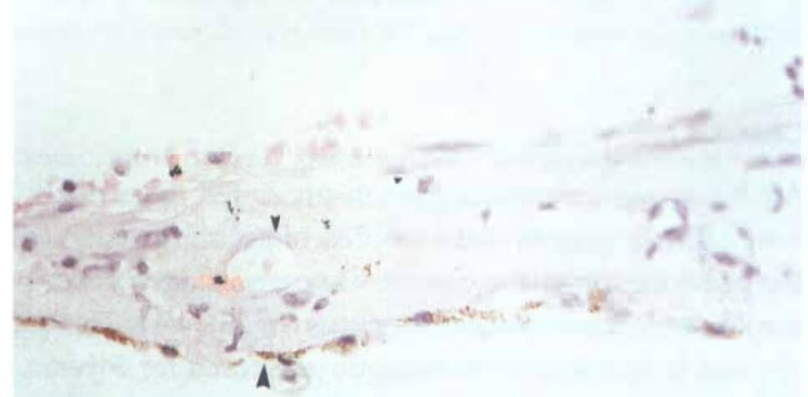

(f)

Fig. 1. Case 1. (a), (b) Post-laser fundus photograph (a) and fluorescein angiogram (b) of the left eye showing diffuse diabetic maculopathy, laser burns and mild macular oedema persisting inferior to fixation. (c), (d) Pre-operative fundus photograph (c) and fluorescein angiogram (d) of the left eye showing subretinal haemorrhage and a large neovascular membrane nasal to the macula and extending subfoveally: (e) Post-operative fundus photograph showing absence of the CNV membrane. ( $f$ ) Light photomicrograph of the fibrovascular membrane. An attenuated layer of retinal pigment epithelium partially lines one surface (large arrow). Vascular channels are also visible (small arrow) (HEE; $\times 190)$. 


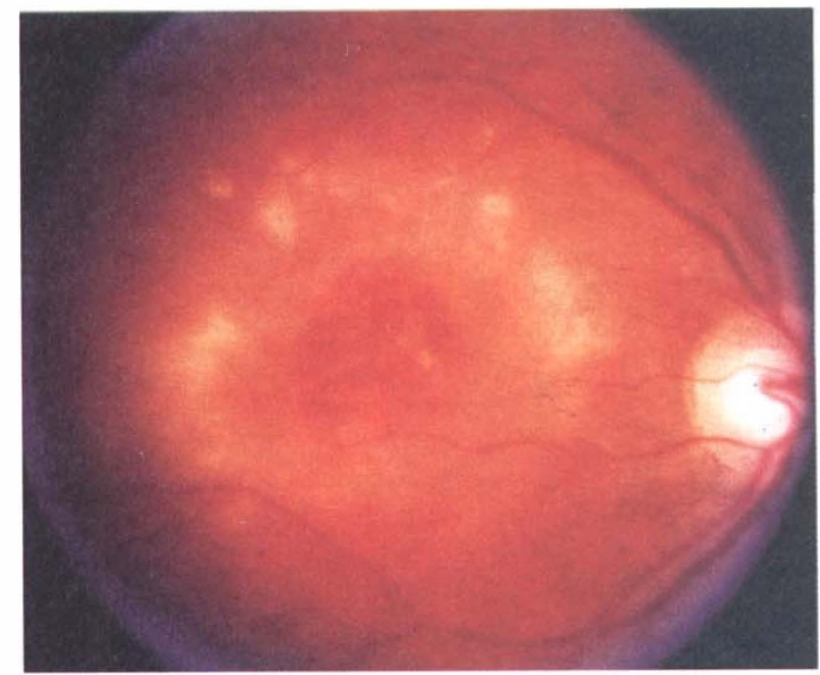

(a)

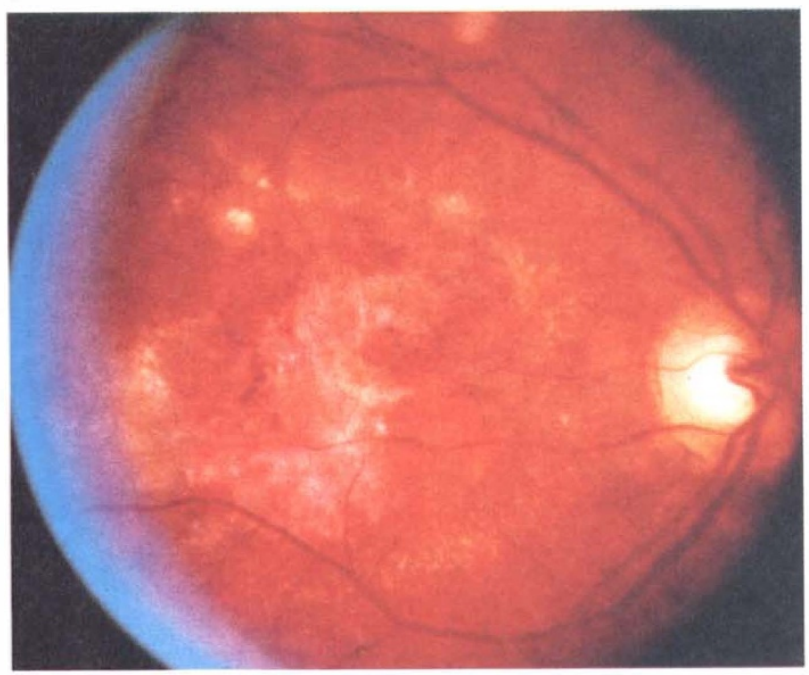

(c)

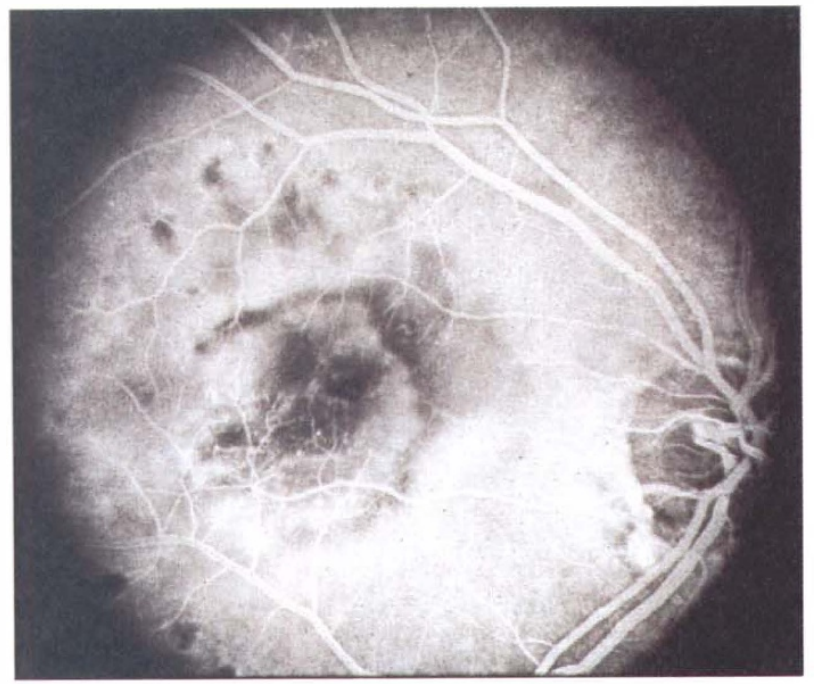

(b)

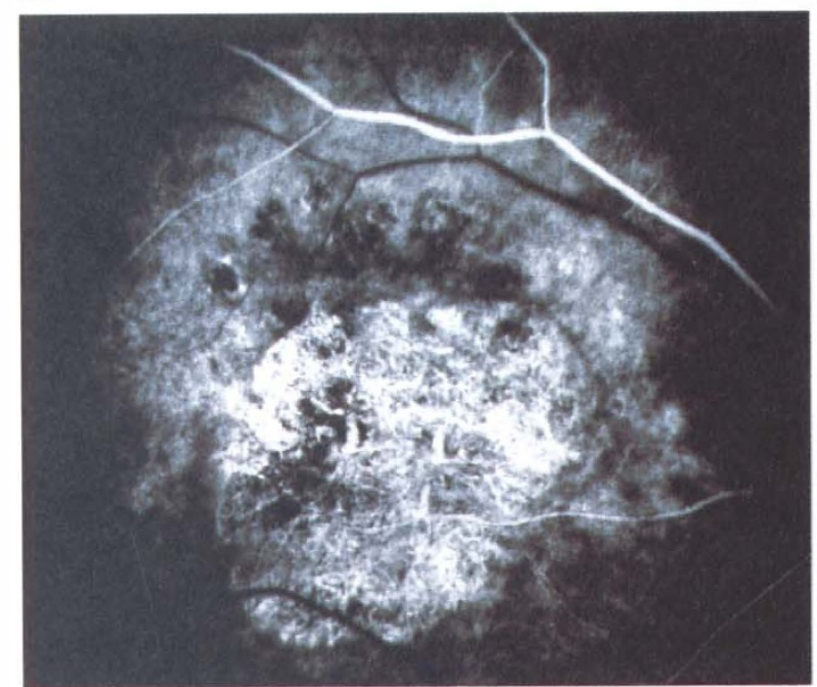

(d)

Fig. 2. Case 2. (a), (b) Pre-operative fundus photograph (a) and fluorescein angiogram (b) of the right eye showing a large subfoveal $C N V$ membrane. (c), (d) Fundus photograph (c) and fluorescein angiogram (d) of the right eye 1 month post-operatively showing that the CNV has been removed, but with associated loss of the retinal pigment epithelium.

\section{Case 3}

A 62-year-old Caucasian woman presented to another hospital with gradual deterioration of her right vision for the previous 4 months. She was known to have noninsulin-dependent diabetes mellitus for 12 years. The left eye had been blind from rubeotic glaucoma for 5 years. Two years previously she had undergone right extracapsular cataract extraction with posterior chamber intraocular lens implantation.

At presentation the right visual acuity was reduced to $6 / 18$ and she had no perception of light in the left eye. She was found to have clinically significant macular oedema and underwent modified grid argon green laser photocoagulation, using $100 \mu \mathrm{m}$ spots, to the temporal part of the macular region. The treatment was described as 'uneventful'.

Two months later she re-presented with rapid deterioration in her right visual acuity. At that time her right visual acuity was $6 / 60$, and the macula was elevated by serous subretinal fluid associated with a subretinal haemorrhage (Fig. 3a, b). Fluorescein angiography showed CNV apparently arising from one of the laser burns and extending under the fovea (Fig. 3c, d). Submacular surgery was recommended.

At surgery the $\mathrm{CNV}$ was relatively easily dislodged from the underlying tissues and following the removal a minute haemorrhage at the edge of the laser scar superotemporal to the fovea appeared, suggesting the site of the original feeder vessel. Histological examination disclosed a typical CNV with abundant fibrous tissue, pigment cells and vascular channels.

Post-operatively the macula was reattached and 6 months later her visual acuity improved to 6/12. A year later, an arrowhead of intraretinal exudates appeared in close proximity to the fovea and fluorescein angiography suggested intraretinal leakage from retinal microaneurysms (Fig. 4). Further focal laser treatment was declined. At 2 years her right visual acuity remained stable at $6 / 12$. 


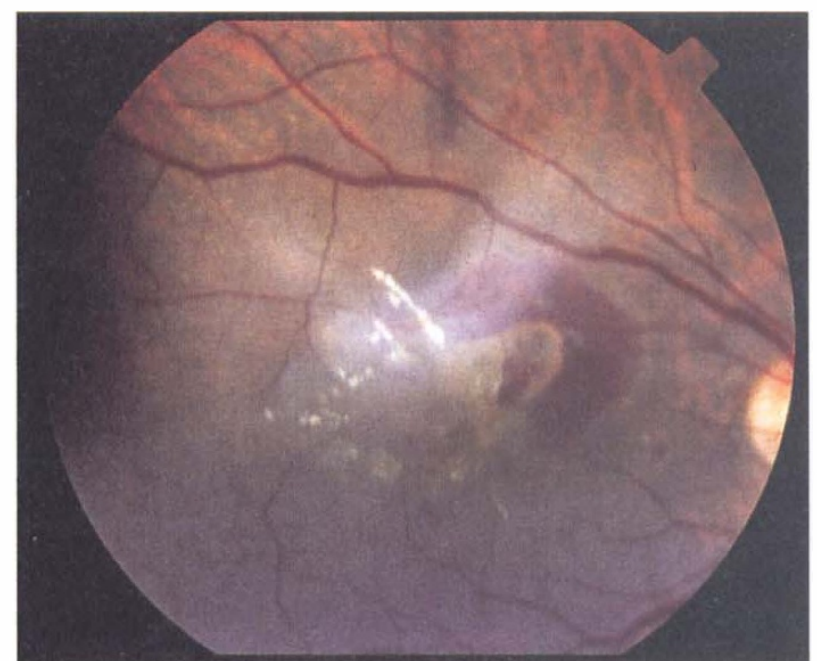

(a)

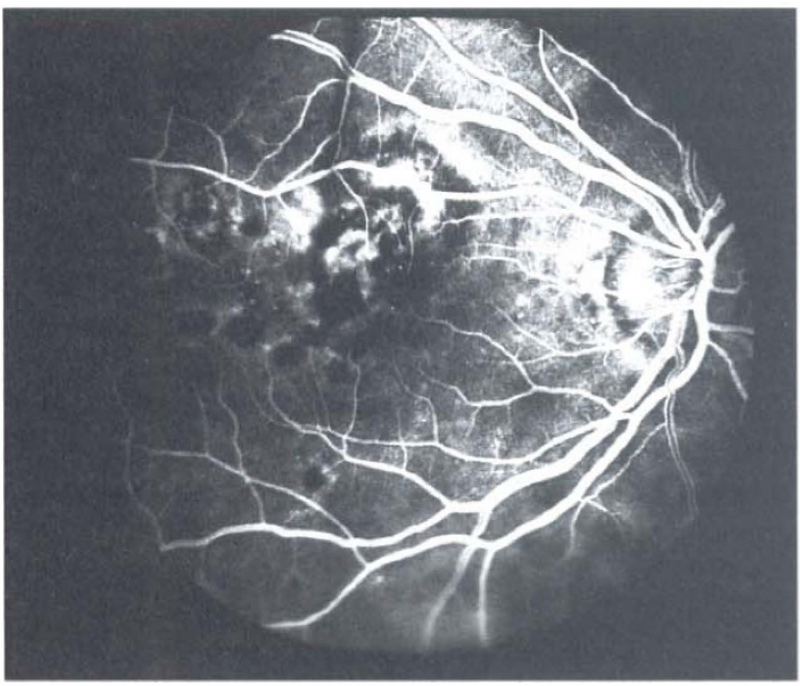

(c)

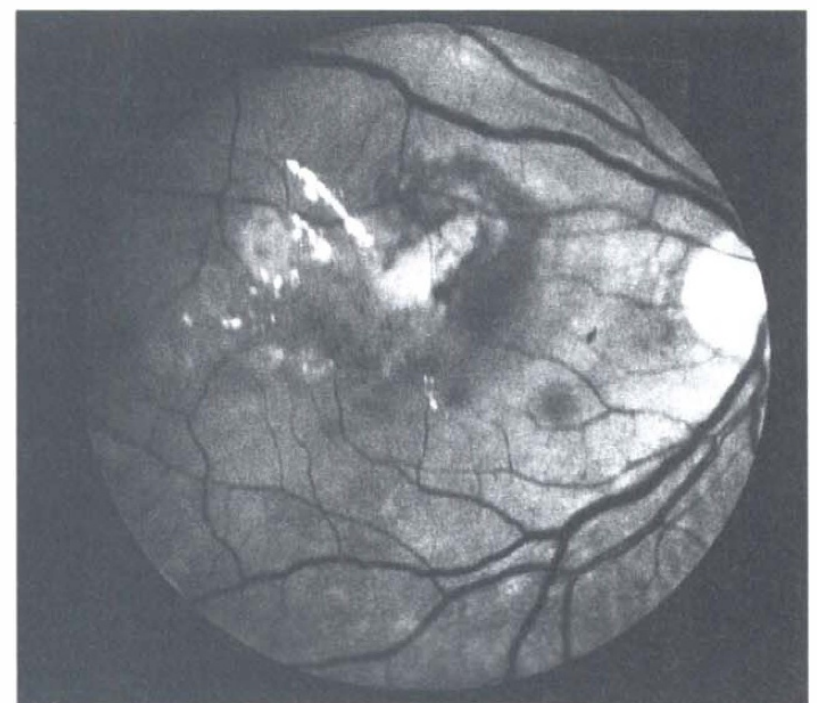

(b)

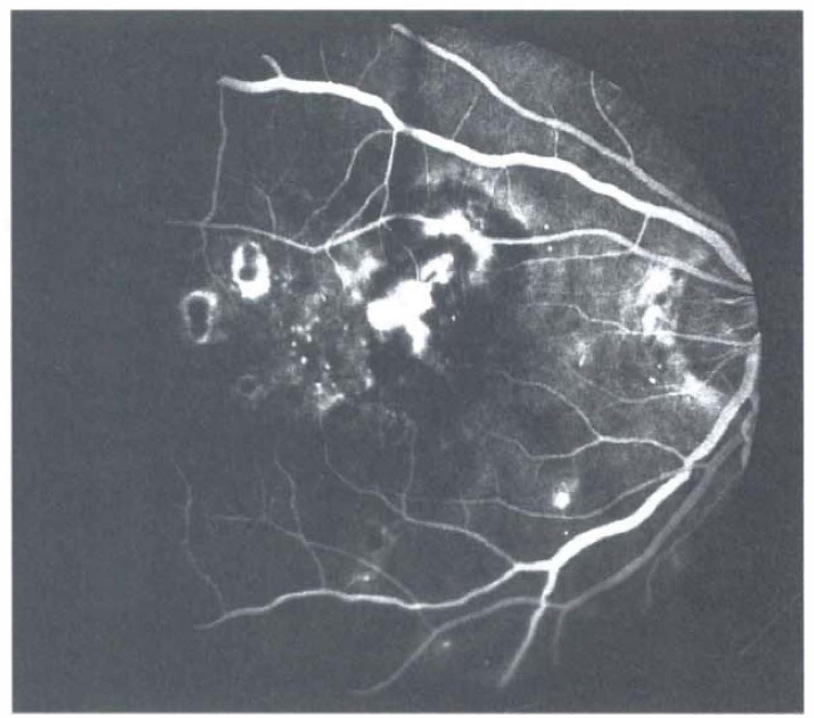

(d)

Fig. 3. Case 3. Pre-operative colour (a) and red-free (b) fundus photographs showing subretinal CNV with associated subretinal haemorrhage and exudate. (c), (d) Pre-operative fluorescein angiogram showing hyperfluorescence in the area of the subfoveal CNV and masking from subretinal blood.

\section{Case 4}

A 60-year-old Caucasian man with adult-onset diabetes mellitus for 17 years presented with a large subretinal haemorrhage in his left eye. His visual acuity was reduced to hand movements (HM). He had undergone macular photocoagulation, at another hospital, 2 years previously but the visual acuity after this procedure is unknown. Fundoscopy showed extensive submacular haemorrhage elevating the retina, with hard exudates, the haemorrhage masking the location of the subretinal neovascular membrane on fluorescein angiography. He underwent pars plana vitrectomy, removal of subretinal blood and removal of subretinal neovascular membrane through a temporal retinotomy. One month postoperatively his vision was $3 / 60$, the macular hard exudates were persisting and an atrophic scar was evident at the retinotomy site. Two months postoperatively, the visual acuity had decreased to counting fingers, and the subretinal choroidal neovascular membrane had recurred with an overlying serous retinal detachment. The patient declined further surgical removal. Although the serous detachment partially subsided with less metamorphopsia by 10 months postoperatively, the visual acuity remained counting fingers, and the patient was subsequently lost to follow-up.

\section{Discussion}

In this paper we report 4 patients who underwent surgical excision of subretinal neovascular membranes which occurred following laser photocoagulation for diabetic maculopathy. Subretinal neovascularisation is a potential cause of severe visual loss in a variety of conditions including age-related macular degeneration, presumed ocular histoplasmosis syndrome, pseudoxanthoma elasticum with angioid streaks, myopia, trauma, following macular laser photocoagulation $^{1-7}$ and may also be idiopathic. 


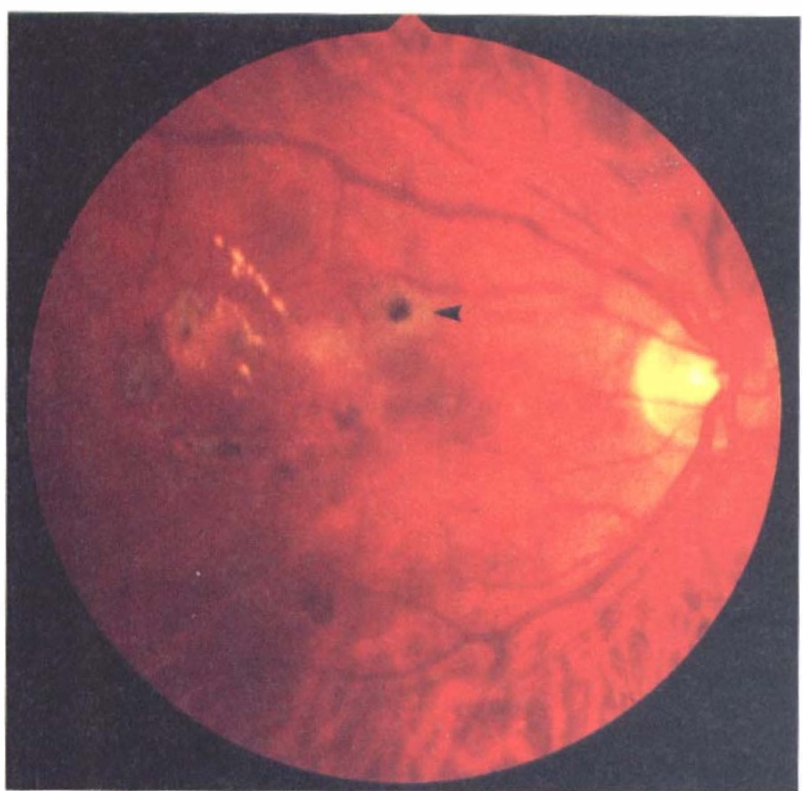

(a)

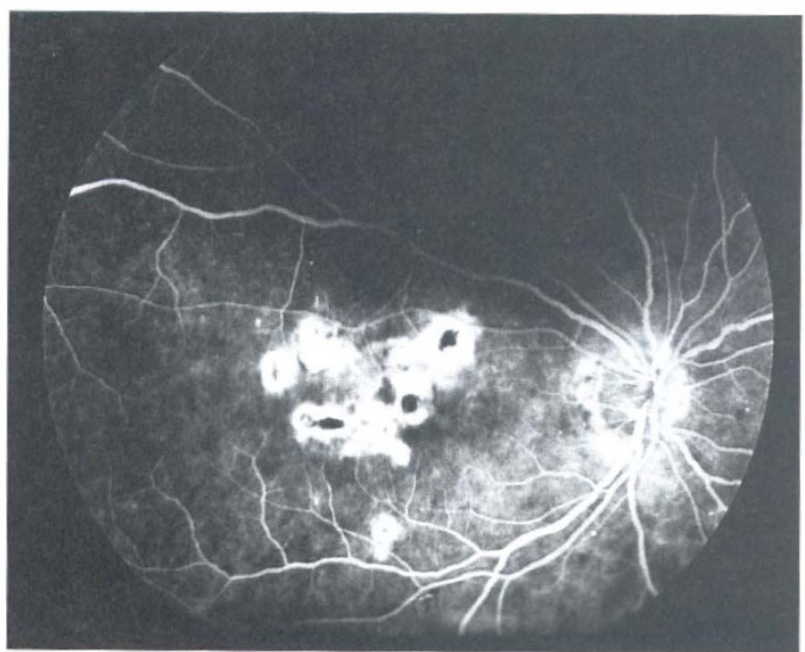

(c)

Diabetic retinopathy is the second most common cause of adult blindness in the Western world. ${ }^{11}$ The benefits of laser photocoagulation in the treatment of diabetic retinopathy, including maculopathy, have been demonstrated in several studies including the Early Treatment of Diabetic Retinopathy Study (ETDRS). ${ }^{12}$ Complications of laser are infrequent and include paracentral scotoma, accidental foveal laser burn, the development of epiretinal membrane (ERM), submacular fibrosis, and the formation of $\mathrm{CNV} \cdot{ }^{13-15}$

Recently, a method of surgical extraction of $\mathrm{CNV}$ was developed in the hope of improving, or at least preserving, central vision. Thomas and Kaplan ${ }^{3}$ found an improvement of visual acuity of 3 Snellen lines following surgical removal of CNV in presumed ocular histoplasmosis syndrome. There have also been suggestions that this may be beneficial in selected cases of age-related macular degeneration, although the results have been generally poor. ${ }^{9,16-18}$ The differences in visual outcome have been attributed by Gass ${ }^{19}$ to the location of the neovascular membrane and the ability to spare the

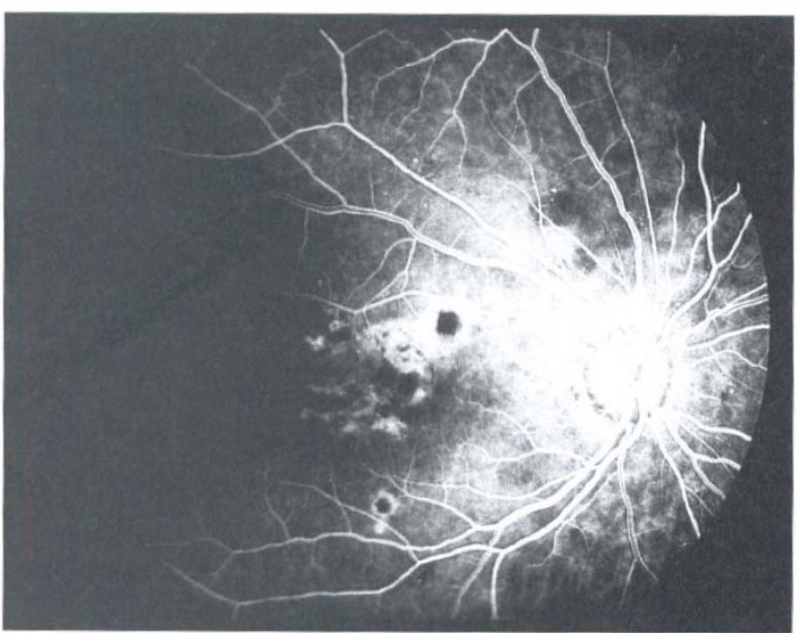

(b)

Fig. 4. Case 3. (a) Post-operative colour photograph showing retinal pigment epithelial changes from prior macular laser treatment and the site of origin of the neovascular membrane (arrow). (b), (c) Postoperative fluorescein angiograms showing hyperfluorescence from retinal pigment epithelial atrophy but no recurrent $\mathrm{CNV}$.

retinal pigment epithelium at the time of surgery. $\mathrm{He}$ proposed a classification of $\mathrm{CNV}$ according to its location. Type $1 \mathrm{CNV}$ is most commonly seen in conditions with diffuse involvement of the RPE, such as age-related macular degeneration, and the neovascular complex underlies and has multiple attachments to the $\mathrm{RPE}$, thus making extraction of the CNV with preservation of the RPE technically impossible. Type 2 $\mathrm{CNV}$ is usually seen in conditions with focal damage of the RPE, such as after POHS or with idiopathic $\mathrm{CNV}$, and it may be possible to remove these membranes while preserving the RPE since the CNV is located in the subneurosensory retinal space and may not be adherent to the RPE. ${ }^{17,20}$

Subfoveal CNV has been reported following previous focal macular laser photocoagulation for diabetic macular oedema. ${ }^{14,21,22}$ Treatment of the CNV membranes with either argon or krypton in the report by Lewis $e t$ al. ${ }^{22}$ was not successful. Subretinal neovascularisation may also occur following grid laser photocoagulation (Brucker et al., unpublished findings). 
All these patients developed subfoveal neovascular membranes and significant visual loss associated with the neovascularisation. None of the patients underwent laser ablation of the neovascularisation or surgical extraction and hence the natural history of the condition is thought to be poor. Indeed, in our present series with at least 9 months follow-up, most of the patients had a reduction in central vision to $6 / 60$ pre-operatively. Previous histopathological reports suggest that the CNV originates from areas of prior photocoagulation that have resulted in disruption or breaks in Bruch's membrane, allowing neovascularisation to extend from the choriocapillaris. $^{23}$

Based on these previous findings, it was felt that surgical removal of neovascularisation may allow stabilisation or improvement in vision in patients with subfoveal neovascular membranes after laser treatment. In this study, we found improvement in vision in the 2 patients who were likely, on their surgical behaviour, to have type 2 membranes. In the third patient the RPE was partially denuded during surgical removal, and consequently she was thought to have a type 1 membrane. This patient had stabilisation of vision with no further active neovascularisation, haemorrhaging or exudation, but did not obtain any improvement with surgery. Interestingly, the fellow eye of this same patient had scattered mild drusen indicating a possible aging disorder of the RPE/Bruch's membrane possibly accounting for the development of the type 1 membrane in the sub-RPE space. There is no clear association between drusen and diabetic retinopathy, and epidemiological studies on this topic are conflicting. ${ }^{24,25}$ However, there is no reason why the two common conditions should not coexist in the same eye. Hence it is possible that $\mathrm{CNV}$ membranes found in diabetics with drusen may be of the type 1 variant, whether focal laser has been applied or not. One would expect laser-related $\mathrm{CNV}$ to be of the focal type 2 variant. Only one patient developed recurrent neovascularisation during the follow-up interval. Interestingly, recurrence rates following surgical extraction of non-age-related $\mathrm{CNV}$ have been reported as being 30\% for type 2 and $60-80 \%$ for type 1 membranes. ${ }^{9}$ The membranes of only 2 of our patients were sent for histopathological examination, and demonstrated the difficulty in differentiating the two subtypes of membranes on excised specimens as compared with enucleated material.

One other recent publication of surgical removal of iatrogenic $\mathrm{CNV}$ reports two cases: one with $\mathrm{CNV}$ arising following focal laser for diabetic macular oedema as in this study, and the other arising following quadrantic scattered photocoagulation for macular oedema following branch retinal vein occlusion. ${ }^{26}$ The $\mathrm{CNV}$ in the latter case was initially treated by focal laser and excision was undertaken later when it recurred. Both patients had improved visual acuity, reduced scotoma size and no recurrence of $\mathrm{CNV}$ in the respectively 1 and 2 year follow-up periods.
Taking our present study along with that of Chen et $a l .{ }^{26}$ we can draw a number of conclusions regarding surgical extraction of laser-related CNV membranes. Firstly, the scar from the previous laser does not prevent surgical access to the CNV and the surgery is technically feasible. Secondly, the visual results may be beneficial, especially if the CNV membrane is of the type 2 variant with only focal involvement of Bruch's membrane (presumed in cases 1 and 3 in our study). A favourable feature on a pre-operative angiogram would be a demonstrable single feeder vessel to the CNV membrane. FInally, CNV recurrence remains a possibility.

\section{References}

1. Ferris FL III, Fine SL, Hyman L. Age-related macular degeneration and blindness due to neovascular maculopathy. Arch Ophthalmol 1984;102:1640-2.

2. Bressler SB, Bressler NM, FIne SL, et al. Natural course of choroidal neovascular membranes within the foveal zone in senile macular degeneration. Am J Ophthalmol 1982;93:157-63.

3. Thomas MA, Kaplan HJ. Surgical removal of subfoveal neovascularisation in the presumed ocular histoplasmosis syndrome. Am J Ophthalmol 1991;111:1-7.

4. Gross JG, King LP, de Juan E Jr, Powers T. Subfoveal neovascular membrane removal in patients with traumatic choroidal rupture. Ophthalmology 1996;103:579-85.

5. Owens SL, Guymer RH, Gross-Jendroska M, Bird AC. Fluorescein angiographic abnormalities after prophylactic macular photocoagulation for high-risk age-related maculopathy. Am J Ophthalmol 1999;127:681-7.

6. Macular Photocoagulation Study Group. Argon laser photocoagulation for senile macular degeneration: results of a randomized clinical trial. Arch Ophthalmol 1982;??:91-918??

7. The Moorfields Macular Study Group. Treatment of senile disciform macular degeneration: a single blind randomized trial by argon laser photocoagulation. Br J Ophthalmol 1992;66:745-53

8. Macular Photocoagulation Study Group. Laser photocoagulation of subfoveal neovascular lesions in agerelated macular degeneration: results of a randomized clinical trial. Arch Ophthalmol 1991;109:1220-31.

9 Eckstein M, Wells JA, Aylward B, Gregor TM. Surgical removal of non-age-related subfoveal choroidal neovascular membranes. Eye 1998;12:775-80.

10. Lambert HM, Capone A Jr, Aaberg TM, et al. Surgical excision of subfoveal neovascular membranes in age-related macular degeneration. Am J Ophthalmol 1992;113:257-62.

11. Government Statistical Service, Office of Population Census and Surveys. Causes of Partial Sight and Blindness in England and Wales. 1990-1991. London: HMSO, 1990.

12. ETDRS Study Group. Early photocoagulation for diabetic retinopathy. ETDRS report no. 9. Ophthalmology 1991;98:766-85.

13. Olk RJ. Modified grid argon (blue-green) laser photocoagulation for diffuse diabetic macular oedema. Ophthalmology 1986;93:938-50.

14. Varley MP, Frank E, Purnell EW. Subretinal neovascularization after focal argon laser for diabetic macular edema. Ophthalmology 1988;95:567.

15. Han DP, Miller WF, Burton TC. Submacular fibrosis after photocoagulation for diabetic macular oedema. Am J Ophthalmol 1992;113:513-21.

16. Berger AS, Kaplan HJ. Clinical experience with the surgical removal of subfoveal neovascular membranes: short-term post-operative results. Ophthalmology 1992;99:969-75. 
17. Thomas MA, Grand G, Williams DF, et al. Surgical management of subfoveal choroidal neovascularization. Ophthalmology 1992;99:952-68.

18. Thomas MA, Dickinson JD, Melberg MS, et al. Visual results after surgical removal of subfoveal choroidal neovascular membranes. Ophthalmology 1994;101:1384-96.

19. Gass JD. Biomicroscopic and histopathologic considerations regarding the feasibility of surgical excision of subfoveal neovascular membranes. Trans Am Ophthalmol Soc 1994;92:91-111.

20. Grossniklaus HE, Gass JD. Clinicopathologic correlations of surgically excised type 1 and type 2 submacular choroidal neovascular membranes. Am J Ophthalmol 1998;126:59-69.

21. Berger AR, Boniuk I. Bilateral subretinal neovascularization after focal argon laser for diabetic macular edema. Am J Ophthalmol 1989;108:88-90.
22. Lewis H, Schacheat AP, Haiman MH, et al. Choroidal neovascularization after laser photocoagulation for diabetic macular edema. Ophthalmology 1990;97:503-10.

23. Pollack A, Korte GE, Weitzner AL, et al. Ultrastructure of Bruch's membrane after krypton laser photocoagulation. Arch Ophthalmol 1986;104:1372-6.

24. Pauleikhoff D, Wormald RP, Wright L, et al. Macular disease in an elderly population. Ger J Ophthalmol 1992;1:12-5.

25. Argan M, Soubrane G, Banlelo, et al. Epidemiological relationship between age-related macular degeneration and diabetes mellitus. Invest Ophthalmol Vis Sci 1990;31:49.

26. Chen CJ, Urban LL, Nelson NC, et al. Surgical removal of subfoveal iatrogenic choroidal neovascular membranes. Ophthalmology 1998;105:1606-11. 